\title{
On Blind Equalization of MIMO Channels
}

\author{
Ye Li and K. J. Ray Liu \\ Electrical Engineering Department and Institute for Systems Research \\ University of Maryland, College Park, MD 20742 \\ E-mail: liye(kjrliu)@src.umd.edu, Fax: (301) 314-9281
}

\begin{abstract}
This paper investigates adaptive blind equalization for multiple-input and multiple-output (MIMO) channels and its application to blind separation of multiple signals received by antenna arrays in communication systems. The performance analysis of the CMA equalizer used in MIMO channels is first presented. Our analysis results indicate that for the MIMO FIR channels satisfying certain conditions, the MIMOCMA FIR equalizer is able to remove the ISI and co-channel interference regardless of the initial setting of the blind equalizer. To recover all input signals simultaneously, a novel MIMO channel blind equalization algorithm is then developed. The global convergence of the new algorithm for MIMO channels is proved. Hence, the new blind equalization algorithm for MIMO channels can be applied to separate and equalize the signals received by antenna arrays in communication systems. Finally, Computer simulations are presented to confirm our analysis and illustrate the performance of the new algorithm.
\end{abstract}

\section{INTRODUCTION}

The use of array signal processing in wireless communications under the framework of spatial division multiple access (SDMA) has been of great interest recently. In such situation, the sensors or antennas may receive a superposition of several signals via many channels from many moving sources. The system can be modeled as a multiple-input/multiple-output (MIMO) system. One of the most crucial problems is not only to separate these signals, but also simultaneously equalize the MIMO channel such that high quality communications can be achieved. The signal separation in other MIMO systems, such as in speech processing, seismic exploration, and the analysis of biological systems, is also an important issue. To separate the signals and at the same time, remove the channel distortion, blind channel equalization techniques have been very effective.

The equalization of MIMO transmission systems is studied in [4] when the MIMO channel impulse response is known. Several iteration algorithms for blind estimation and separation of MIMO FIR channels have been developed in [9]. However, there is no proof on the global convergence of those iteration algorithms. The blind identification and multiple signal separation algorithms based on higher-order statistics have been presented in [6], [10], [11]. However, there is no report on the adaptive blind equalization for MIMO channels. The CMA [1], [7], [8] is one of the most popular algorithms used in single-input/signal-output (SISO) systems. It can also be used in single-input/multiple-output (SIMO) systems [3]. An interesting question is how effective the CMA is when it is used in MIMO systems, such as in mobile communications, to remove intersymbol interference (ISI), co-channel in-

* The work was supported in part by the NSF grants MIP9309506 and MIP9457397. terference (CCI) or adjacent-channel interference (ACI). This paper first investigates the possibility of the use of CMA blind equalizer in MIMO channels. Our analysis demonstrates that the CMA blind equalizer is able to recover one of the input signals, suppress the rest of signals. Furthermore, under certain condition, the CMA FIR equalizer, regardless of its initial setting, can perfectly recover one of the input signals from the outputs of the MIMO FIR channels. Therefore, the CMA can be used in mobile communication systems to remove ISI, CCI, and ACI. Then, we develop a new adaptive blind equalization algorithm for MIMO channels, to simultaneously recover all the input signals and at the same time to remove the ISI.

\section{Blind Equalization for Mimo Channels}

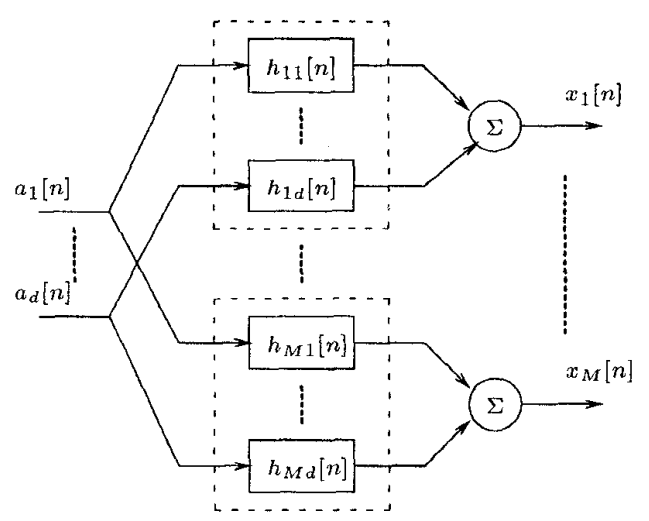

(a)

Fig. 1. Multiple-input and multiple-output channel

Antenna arrays can be used in mobile communication systems to improve the communication quality and increase communication capacity. The antenna array received the superposition of several wide-band signals can be modelled as an MIMO system shown in Figure 1. The $d$ complex sequences $a_{1}[n], \cdots, a_{d}[n]$ are sent through different channels with impulse responses $h_{i j}[n]$ for $i=1, \cdots, M$ and $j=1, \cdots, d$ $(d \leq M)$. We will assume in this paper that the input sequences satisfy

$$
E\left\{a_{i}[n]\right\}=E\left\{a_{i}^{2}[n]\right\}=0,
$$

and

$$
2 m_{2}^{2}-m_{4}>0
$$


where

$$
m_{2} \triangleq E\left\{\left|a_{i}[n]\right|^{2}\right\}, m_{4} \triangleq E\left\{\left|a_{i}[n]\right|^{4}\right\}
$$

If we define the output vector $\mathbf{x}[n]$, the input vector $\mathbf{a}[n]$, and the channel impulse response matrix $H[n]$ respectively as

$$
\mathbf{x}[n] \triangleq\left(\begin{array}{c}
x_{1}[n] \\
\vdots \\
x_{M}[n]
\end{array}\right), \mathbf{a}[n] \triangleq\left(\begin{array}{c}
a_{1}[n] \\
\vdots \\
a_{d}[n]
\end{array}\right)
$$

and

$$
H[n] \triangleq\left(\begin{array}{ccc}
h_{11}[n] & \ldots & h_{1 d}[n] \\
\vdots & \vdots & \vdots \\
h_{M 1}[n] & \ldots & h_{M d}[n]
\end{array}\right)
$$

then the channel output vector $\mathbf{x}[n]$ can be expressed as

$$
\mathbf{x}[n]=H[n] * \mathbf{a}[n],
$$

where $*$ denotes the convolution of the matrix (or vector) sequences. For general matrix sequences $\left(a_{i j}[n]\right)$ and $\left(b_{i j}[n]\right)$, their convolution is defined as

$$
\left(a_{i j}[n]\right) *\left(b_{i j}[n]\right) \triangleq\left(\sum_{k} a_{i k}[n] * b_{k j}[n]\right) .
$$

Equation (6) can also be written in $Z$-transform as

$$
\mathbf{x}(z)=H(z) \mathbf{a}(z),
$$

where $\mathbf{x}(z), \mathbf{a}(z)$ and $H(z)$ are the $Z$-transform of $\mathbf{x}[n], \mathbf{a}[n]$ and $H[n]$, respectively. For MIMO FIR channels, $H(z)$ is a polynomial matrix.

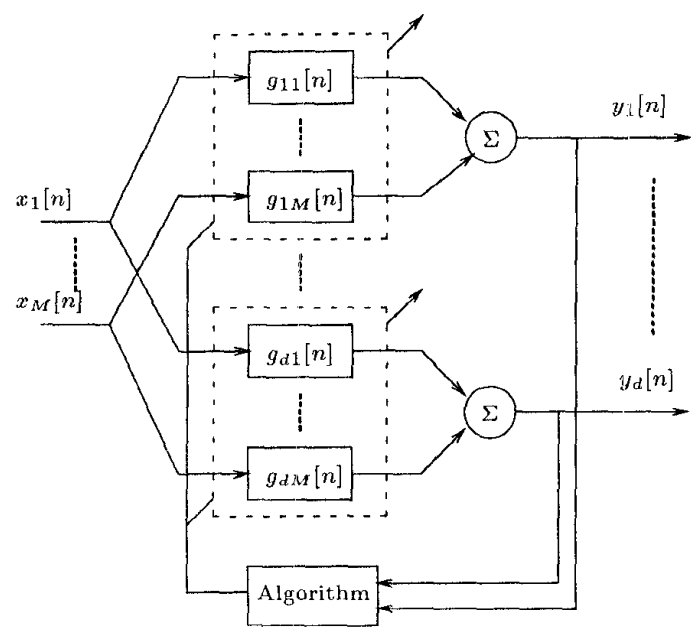

(b)

Fig. 2. Adaptive blind multiple-channel equalizer.

To recover the input signal $\mathbf{a}[n]$, a linear channel equalizer is applied to the channel output $\mathbf{x}[n]$ as in Figure 2, whose objective is to achieve distortionless recovery. That is, to find $G[n]$ such that

$$
G[n] * H[n]=\delta[n] I_{d},
$$

or simply

$$
G(z) H(z)=I_{d},
$$

where $I_{d}$ is a $d \times d$ identity matrix and $G[n]$ is the equalizer matrix defined as

$$
G[n] \triangleq\left(\begin{array}{ccc}
g_{11}[n] & \cdots & g_{1 M}[n] \\
\vdots & \cdots & \vdots \\
g_{d 1}[n] & \cdots & g_{d M}[n]
\end{array}\right),
$$

and $G(z)$ is the $Z$-transform of $G[n]$. Initially, we may take the filters in Figure 2 as being bounded-input and boundedoutput (BIBO) stable and potentially non-causal (doubleinfinite) so as to deal with MIMO channels of non-causal inverse. In blind equalization, the original sequences $a_{i}[n] \in \mathcal{A}_{i}$ for $i=1, \cdots, d$ are unknown to the receivers except for their statistical properties over the known alphabet sets $\mathcal{A}_{i}$. Usually, the signal constellations $\mathcal{A}_{1}=\mathcal{A}_{2}=\cdots=\mathcal{A}_{d}$ are symmetric such that the statistics of the input signals $a_{i}$ for $i=1, \cdots, d$ reflects the same symmetry. Thus, the recoverable signals from blind equalization will similarly subject to a phase ambiguity and a permutation ambiguity. Therefore, the best possible result of blind MIMO equalizers would be

$$
G(z) H(z)=P D(z),
$$

where $P$ is a $d \times d$ permutation matrix and $D(z)$ is a diagonal matrix defined as

$$
D(z)=\operatorname{diag}\left\{e^{\jmath \theta_{1}} z^{-n_{1}}, \cdots, e^{\jmath \theta_{d}} z^{-n_{d}}\right\}
$$

where $\theta_{i} \in[-\pi, \pi]$ and $n_{i}$ is an integer for $i=1, \cdots, d$. The equalizers with $G(z)$ satisfying (12) are called the distortionless recovery equalizer for channel $H(z)$. It is obvious that the distortionless recovery equalizer for a given MIMO channel is not necessarily unique.

Not all channels have a BIBO stable distortionless recovery equalizer. A channel is said to satisfy the distortionless recovery condition if there exists a $\mathrm{BIBO}$ stable distortionless recovery equalizer for such channel. For MIMO channels, the following theorem gives a necessary and sufficient condition for the existence of BIBO stable distortionless recovery equalizers.

Theorem 1: A BIBO stable, linear, and distortionless recovery equalizer for an MIMO channel exists if and only if

$$
\operatorname{det}\left(H^{H}\left(e^{\jmath \omega}\right) H\left(e^{\jmath \omega}\right)\right) \neq 0, \text { for all } \omega \in[-\pi, \pi] .
$$

From Theorem 1, a necessary condition for an MIMO channel to have distortionless recovery equalizer is $M \geq d$, that is, the number of channel outputs is no less than the number of channel inputs. In what follows, we will always assume that the discussed MIMO channels satisfy the distortionless recovery condition.

\section{ThE CMA IN FIR MIMO ChanNels}

In this section, we will investigate the performance of the CMA equalizer used in MIMO channels. The MIMO-CMA 


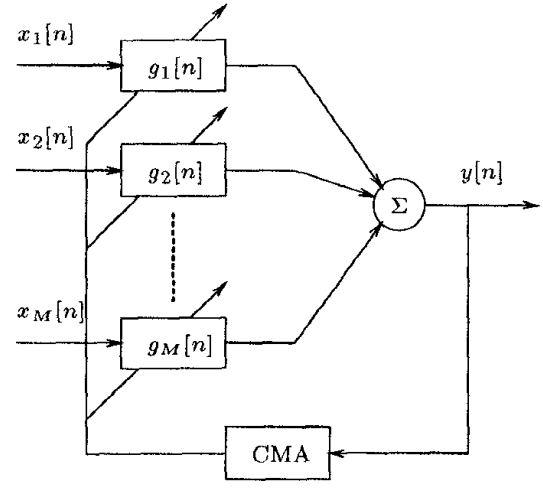

Fig. 3. The MIMO-CMA blind equalizer.

blind equalizer discussed in this section is illustrated in Figure 3. After each channel output, a linear BIBO stable filter is used. The filter coefficients are adjusted to minimize the Godard cost function $[1],[7],[8]$

$$
C(y[n])=\frac{1}{4} E\left\{\left(|y[n]|^{2}-r\right)^{2}\right\},
$$

where $r$ is the dispersion constant defined as

$$
r=\frac{m_{4}}{m_{2}}
$$

In practice, most of the MIMO channels can be approximated as FIR MIMO channels. The length of the impulse response of the equalized system is $L+K-1$ if an MIMOCMA FIR equalizer with length $K$ is used for the MIMO FIR channel.

For the CMA FIR equalizers used in MIMO FIR channels, We can prove the following global convergence theorem.

Theorem 2: For an MIMO FIR channel of length $L$, if $H(z)$ is irreducible with $H[L-1]$ being of full rank, then any MIMO-CMA FIR blind equalizer with length $K \geq\left\lceil\frac{(L-1) d}{M-d}\right\rceil$ can achieve global convergence regardless of the its initial setting.

The above theorem illustrates a very nice convergence property of the MIMO-CMA FIR equalizer used in MIMO channels. It indicates that the MIMO-CMA FIR equalizer can recover one of the input signals, remove ISI, and suppress CCI and ACI if the channel satisfies certain conditions.

\section{A New Blind Equalization Algorithm for MIMO CHANNELS}

We have studied the convergence of the CMA blind equalizer used in MIMO channels in the previous section and have shown that the output of the MIMO-CMA blind equalizer can recover one of the input signals and suppress the interference from the rest of the input signals. In this section, we will propose a new blind equalization algorithm, which can recover all input signals simultaneously. Without lost of generality, we will assume $d=2$ in this section. The algorithm developed in this section can be easily extended to $d>2$ case.

\section{A. Algorithm Development}

Consider the blind equalizer shown in Figure 2. If we adjust the equalizer parameters for each individual channel to minimize the Godard cost function in (15), then according to the analysis of Section III, the equalizer outputs $y_{1}[n]$ and $y_{2}[n]$ will be one of the input signals but we do not know which of the input signals. Note that $y_{1}[n]$ and $y_{2}[n]$ are either the same as or different from each other depending on the initial setting of the equalizer. Hence, to develop an algorithm that can simultaneously recover all input signals, we may have to modify the Godard cost function.

The new cost function for the adaptive blind equalization of MIMO channels is given as follows:

$$
C_{M I M O} \triangleq C\left(y_{1}[n]\right)+C\left(y_{2}[n]\right)-c_{o} K\left(y_{1}, y_{2}\right),
$$

where $c_{o} \geq m_{4} /\left(2 m_{2}^{2}-m_{4}\right)$, and $K\left(y_{1}, y_{2}\right)$ is a functional of $y_{1}(k)$ and $y_{2}(k)$ for all $k \leq n$ defined as

$$
\begin{aligned}
K\left(y_{1}, y_{2}\right) \triangleq \quad & \frac{1}{2} \sum_{k=-\infty}^{-1} \operatorname{Cum}\left(y_{1}[n], y_{1}^{*}[n], y_{2}[n+k], y_{2}^{*}[n+k]\right) \\
& +\frac{1}{2} \sum_{k=0}^{\infty} \operatorname{Cum}\left(y_{1}[n-k], y_{1}^{*}[n-k], y_{2}[n], y_{2}^{*}[n]\right)
\end{aligned}
$$

with $\operatorname{Cum}\left(y_{1}, y_{1}^{*}, y_{2}, y_{2}^{*}\right)$ being the cumulant of random complex variables $y_{1}, y_{1}^{*}, y_{2}$ and $y_{2}^{*}$ defined as

$$
\begin{gathered}
C u m\left(y_{1}, y_{1}^{*}, y_{2}, y_{2}^{*}\right) \triangleq E\left\{\left|y_{1}\right|^{2}\left|y_{2}\right|^{2}\right\}-E\left\{\left|y_{1}\right|^{2}\right\} E\left\{\left|y_{2}\right|^{2}\right\} \\
-\left|E\left\{y_{1} y_{2}^{*}\right\}\right|^{2}
\end{gathered}
$$

for random variables $y_{i}$ satisfying

$$
E\left\{y_{i}\right\}=E\left\{y_{i}^{2}\right\}=0 \text { for } i=1,2 .
$$

It is the last term in (17) that makes it possible for the equalizer to converge to distinct input signals at each output of the equalizer.

Using the stochastic gradient method to search for minimum points of the new cost function, we can implement the new algorithm as

$$
\begin{aligned}
g_{j m}^{(n)}[k]= & g_{j m}^{(n-1)}[k] \\
& -\mu\left(\left(\left|y_{j}[n]\right|^{2}-r\right) y_{j}[n]-c_{o} z_{j}[n]\right) x_{m}^{*}[n-k],
\end{aligned}
$$

for $j=1,2$ and $m=1, \cdots, M$, where $\mu$ is a small step-size, $g_{j m}^{(n)}[k]$ is the $k$-th parameter of the $j m$-th filter after the $n$-th iteration, and $z_{i}[n]$ 's are given by

$$
\begin{aligned}
z_{1}[n]= & \sum_{l=0}^{\infty}\left\{\left|y_{2}[n-l]\right|^{2} y_{1}[n]-E\left\{\left|y_{2}[n-l]\right|^{2}\right\} y_{1}[n]\right. \\
& \left.\left.-E\left\{y_{1}[n] y_{2}^{*}[n-l]\right\} y_{2}[n-l]\right)\right\}, \\
z_{2}[n]= & \sum_{l=0}^{\infty}\left\{\left|y_{1}[n-l]\right|^{2} y_{2}[n]-E\left\{\left|y_{1}[n-l]\right|^{2}\right\} y_{2}[n]\right. \\
& \left.-E\left\{y_{2}[n] y_{1}^{*}[n-l]\right\} y_{1}[n-l]\right\} .
\end{aligned}
$$

If the ensemble average in the above expressions is substituted by empirical average as in [5], the resulting algorithm can be expressed as

$$
\begin{aligned}
g_{j m}^{(n)}[k]= & g_{j m}^{(n-1)}[k] \\
& -\mu\left(\left(\left|y_{j}[n]\right|^{2}-r\right) y_{j}[n]-c_{o} z_{j}[n]\right) x_{m}^{*}[n-k],
\end{aligned}
$$




$$
\begin{aligned}
& z_{1}[n]=\sum_{l=0}^{\infty}\left(\left|y_{2}[n-l]\right|^{2} y_{1}[n]-r_{2}^{(n)}[l] y_{1}[n]-r_{12}^{(n)}[l] y_{2}[n-l]\right), \\
& z_{2}[n]=\sum_{l=0}^{\infty}\left(\left|y_{1}[n-l]\right|^{2} y_{2}[n]-r_{1}^{(n)}[l] y_{2}[n]-r_{21}^{(n)}[l] y_{1}[n-l]\right),
\end{aligned}
$$

where

$$
\begin{gathered}
r_{2}^{(n)}[l]=(1-\epsilon) r_{2}^{(n-1)}[l]+\epsilon\left|y_{2}[n-1]\right|^{2}, \\
r_{1}^{(n)}[l]=(1-\epsilon) r_{1}^{(n-1)}[l]+\epsilon\left|y_{1}[n-1]\right|^{2}, \\
r_{12}^{(n)}[l]=(1-\epsilon) r_{12}^{(n-1)}[l]+\epsilon y_{1}[n] y_{2}^{*}[n-l],
\end{gathered}
$$

and

$$
r_{21}^{(n)}[l]=(1-\epsilon) r_{21}^{(n-1)}[l]+\epsilon y_{2}[n] y_{1}^{*}[n-l] .
$$

with $\epsilon$ being the forgetting factor.

Remark: We have developed MIMO adaptive blind equalization algorithm for $d=2$. For $d>2$ case, the cost function $C_{M I M O}$ can be extended to

$$
C_{M I M O}=\sum_{i=1}^{d} C\left(y_{i}[n]\right)-c_{o} \sum_{i, j=1}^{d} K\left(y_{i}, y_{j}\right) .
$$

The global convergence and local convergence properties discussed below can be similarly generalized to this case.

\section{B. Convergence}

Directly from the cost function of the new equalization algorithm, we are able to prove global convergence of the new algorithm as the following theorem.

Theorem 3: The MIMO blind equalizer using the cost function defined in (17) will converge to one of its global minimum regarless of its initial setting, if the equalizer length and the channel parameters satisfy one of the following two conditions:

1. The MIMO channel satisfies distortionless recovery condition and an infinite-length MIMO equalizer is used, or

2. $H(z)$, the Z-transform of the impulse response of the MIMO channel of length $L$, is an irreducible polynomial matrix with $H[L-1]$ being nonsingular, and the length of the equalizer $K \geq\left\lceil\frac{(L-1) d}{M-d}\right\rceil$.

From Theorem 3 , the new algorithm is able to recover all input signals simultaneously. Furthermore, the MIMO FIR channel satisfying certain condition can be perfectly equalized by MIMO FIR equalizers employing the new algorithm.

Similar to the blind equalizers for SISO channels, the new MIMO blind equalizer algorithm also suffers from the local convergence if the MIMO equalizers or channels under consideration does not satisfy the conditions in Theorem 3 . Similar to [2], we can construct some example to illustrate this phenomena.

\section{Computer Simulations}

In order to confirm the analysis results and illustrate the effectiveness of the proposed algorithm, we present two computer simulation examples.
In our simulations, the input signals $a_{i}[n]$ are independent of each other for any different $i$ nad $n$, and they are uniformly distributed over $\{ \pm 1, \pm J\}$. The channel noise is complex white Gaussian with zero mean and variance determined by the signal-to-noise ratio $(S N R)$.

\section{A. Convergence of the MIMO-CMA blind equalizer}

In this simulation example, we have $d=2$ and $M=3$. The channel impulse response is given by

$$
H[0]=\left(\begin{array}{cc}
-1.9522 & -0.5706 \\
-0.5666 & 0.4246 \\
-1.1293 & 0.7666
\end{array}\right)
$$

and

$$
H[1]=\left(\begin{array}{cc}
1.0691 & -1.8841 \\
-0.7926 & 0.0598 \\
0.3569 & -0.2744
\end{array}\right)
$$

and $S N R=30 \mathrm{~dB}$.

An MIMO-CMA FIR equalizer is used for the MIMO FIR channel. The length of the equalizer is $K=3$ with initial setting $g_{m}[n]=\delta[m-1, n-1]$ and the step-size $\mu=0.0005$. Figure 4 is the impulse responses of the equalized system after 10,000 iterations. From this figure, the MIMO-CMA FIR equalizer is able to recover the second input signal, remove the ISI and suppress the first signal. Figure 5 illustrates the changing of IT during iterations. The IT here is an index of measurement for intersymbol interference and co-channel interference defined as

$$
I T=\frac{\sum_{i, n}\left|s_{i}[n]\right|^{2}-\max _{i, n}\left|s_{i}[n]\right|^{2}}{\max _{i, n}\left|s_{i}[n]\right|^{2}} .
$$

The simulation results in these two figures confirm Theorem 2 .
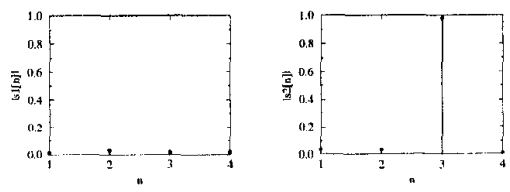

Fig. 4. The impulse response of the equalized system after 10,000 iterations.

\section{B. Convergence of the new MIMO blind equalizer}

This is a 2-input/4-output FIR channel with $S N R=30 \mathrm{~dB}$. The length of the equalizer used in our simulation is 20 with initial setting $g_{11}[n]=g_{22}[n]=\delta[n-10]$. The step-size is $\mu=0.0001$ and the forgetting factor is given by $\epsilon=0.01$. 


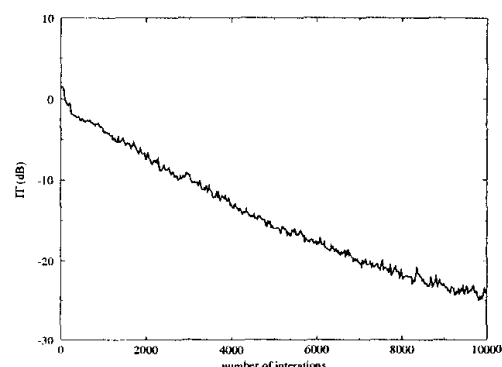

Fig. 5. Convergence of MIMO-CMA blind equalizer.

Figure 6 is the impulse response $s_{i j}[n]$ of the equalized system after 20,000 iterations. Figure 7 illustrates the intersymbol and co-channel interference of the equalizer outputs, which is defined as

$$
I T_{j}=\frac{\sum_{i, n}\left|s_{i j}[n]\right|^{2}-\max _{i, n}\left|s_{i j}[n]\right|^{2}}{\max _{i, n}\left|s_{i j}[n]\right|^{2}} .
$$

According to Figure 6, the two input signals are separated. The first equalizer recovers the first signal and the second recovers the second signal. From the simulation results, our new blind equalization algorithm can simultaneously reconstruct the input signals and remove intersymbol and co-channel interference effectively.
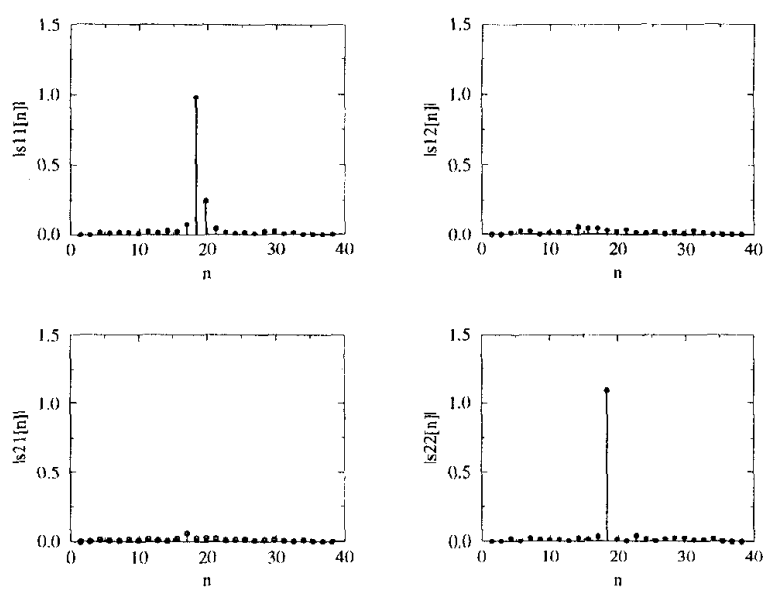

Fig. 6. The impulse response of the equalized system after 20,000 iterations

\section{Conclusions}

This paper investigated the blind equalization of MIMO channels for multiple signals separation. We studied the convergence of the CMA blind equalizer used in MIMO channels.
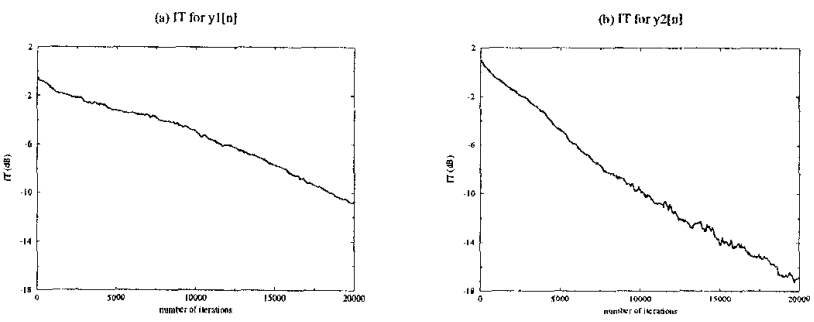

Fig. 7. Convergence of the new algorithm for the MIMO channel

We demonstrated that the CMA blind equalizer is able to recover one of input signals and suppress the rest of input signals. Hence, by proper initialization, the CMA blind equalizer can be used in mobile communication systems to recover the desire signal, remove intersymbol interference, and suppress co-channel interference and adjacent channel interference. To recover all input signals simultaneously, we proposed a new blind equalization algorithm to separate all the input signals and at the same time equalize the MIMO channel. The global convergence of the new algorithm is illustrated theoretically and by computer simulation. The proposed algorithm not only can be applied in multiple signals separation in array processing, but also can be used in diverse fields of engineering including speech proccssing, data communication, sonar array processing, and in the analysis of biological systems.

\section{REFERENCES}

[1] D. N. Godard, "Self-recovering equalization and carrier tracking in two-dimensional data communication systems," IEEE Trans, on Comm., COM-28:1867-1875, 1980.

[2] Y. Li and Z. Ding, "Convergence analysis of finite length blind adaptive equalizers,' IEEE Transactions on Signal Processing. vol.43, pp2120-2129, September 1995.

[3] Y. Li and Z. Ding, 'Global convergence of fractionally spaced Godard equalizer,' The 26th Asiloma Conference on Signal, Systems 8 Computers, California, October 1994

[4] B. R. Petersen and D. D. Falconer "Suppression of adjacentchannel, co-channel, and intersymbol interference by equalizers and linear combiners," IEEE Transactions on Comm., COM-42:3109. 3118, Dec. 1994

[5] O. Shalvi and E. Weinstein, "New criteria for blind deconvolution of non-minimum phase systems (channels)," IEEE Transactions on Information Theory, IT-36:312-321, March 1990.

[6] E. Weinstein, A. Swami, G. Giannakis, and S. Shamsunder, "Multichannel ARMA processes," IEEE Transactions on Signal Processing, SP-42:898-913, April 1994

[7] J. R. Treichler and B. G. Agee "A new approach to multipath correction of constant modulus signals", IEEE Trans. on Acoustics, Speech and Signal Processing, ASSP-31:349-372, 1983.

[8] J. R. Treichler and M.G. Larimore, "New processing techniques based on the constant modulus adaptive algorithm", IEEE Trans. on Acoustics, Speech and Signal Processing, ASSP-33:420-431, 1985 .

[9] A. van der Veen, S. Talwar, and A. Paulraj, "Blind estimation of multiple digital signals transmitted over FIR channels," IEEE SP Lett., vol.2, May 1995.

[10] E. Weinstein, A. V. Oppenheim, M. Feder, and J. R. Buck, "Iterative and sequential algorithms for multisensor signal enhancement," IEEE Transactions on Signal Processing, SP-42:846-859, April 1994.

[11] D. Yellin and E. Weinstein, "Criteria for multichannel signal separation," IEEE Transactions on Signal Processing, SP-42:2158-2168, August 1994. 